\title{
On the Differential Forms of the First Kind on Algebraic Varieties II.
}

\author{
Shoji Korzumi.
}

(Received Oct. 30, 1950)

We shall give some supplementary remarks to my previous paper on the same subject ${ }^{1)}$. As in Weil's definition, we shall call differential forms to be of the first kind on a Variety $\boldsymbol{U}$, when they are finite at every simple Point on every Variety birationally equivalent to $\boldsymbol{U}$. This definition is equivalent to my previous one in $[K]$, if $\boldsymbol{U}$ has a birationally equivalent model which is a complete Variety without singularities.

1. We shall prove the following theorem as an extension of the theorem 2 in $[K]$.

THEOREM 1. Let $\omega$ be a differential form of the first kind of degreer on a Product-Variety $\boldsymbol{U} \times \boldsymbol{V}$, then we have the following expression.

$$
\omega=\sum \sigma_{i} \tau_{i}
$$

where $\sigma_{i}, \tau_{i}$ are, respectively, differential forms of the first kind on $\boldsymbol{U}, \boldsymbol{V}$, of degree $d_{i} ; r-d_{i}$. Moreover, if $\boldsymbol{\omega}, \boldsymbol{U}$ and $\boldsymbol{V}$ have a common field $k$ of definition which is perfect, $\sigma_{i}, \tau_{i}$ are defined over $k$.

PROOF. Without loss of generality we may suppose that $\omega, \boldsymbol{U}$ and $\boldsymbol{V}$ are defined over a perfect field $k$. Let $\boldsymbol{P}$ and $\boldsymbol{Q}$ be independent generic Points over $k$, of $\boldsymbol{U}$ and $\boldsymbol{V}$, respectively. If $(t)$ and $(u)$ are respectively, sets of uniformizing parameters at $\boldsymbol{P}$ and $\boldsymbol{Q}$, on $\boldsymbol{U}$ and $\boldsymbol{V}$, then

$$
\begin{aligned}
\boldsymbol{\omega} & =\sum_{(i, j)} z_{i_{1}, \ldots, i_{s} ; j_{1}, \ldots, i_{r-s}} d t_{i_{1}} \cdots d t_{i_{s}} d u_{j_{1}} \cdots d u_{j_{r-s}} \\
& =\sum_{j}\left(\sum_{i} z_{(i, j)} d t_{i_{1}} \cdots d t_{i_{s}}\right) d u_{j_{1}} \cdots d u_{j_{r-s}}
\end{aligned}
$$

where $z i_{1}, \cdots, i_{s} ; j_{1}, \cdots, j_{r-s}$ are contained in $k(\boldsymbol{P}, \boldsymbol{Q})$ and $(i, j)$ means $i_{1}, \cdots, i_{s} ; j_{1}, \cdots, j_{r-s}$. If we consider $\sum_{i} z_{(i, j)} d t_{i_{1}} \cdots d t_{i_{s}}$ as defined on $\boldsymbol{U}$ over the field $k(\boldsymbol{Q})$, they are of the first kind.

1) Journal of the Mathematical Society of Japan Vol. 1, No. 3, 1949. This note will be denoted by $[K]$, and we shall use the same terminologies and notations as in $[K]$. 
Therefore from the theorem 4 in $[K]^{2)}$, we obtain

$$
\sum_{i} z_{(i, j)} d t_{1} \cdots d t i_{s}=\sum u_{(j) k} \sigma_{k}
$$

where $u_{(j) k}$ are contained in $k(\boldsymbol{Q})$, and $\sigma_{k}$ are the differential forms of the first kind on $\boldsymbol{U}$ defined over $k$.

So it follows

$$
\omega=\sum \sigma_{i} \tau_{i}
$$

where $\sigma_{i}$ are as above and $\tau_{i}$ are the differential forms on $\boldsymbol{V}$ over $k$. We shall denote $d t_{i} \cdots d t_{s}$ by $T_{\alpha}(\alpha=1, \cdots, \cdots, N)$, and may take the following base $\left\{\sigma_{i, j}\right\}$ of the vector space over $k$ spanned by $\left\{\sigma_{i}\right\}$

$$
\begin{aligned}
& \sigma_{0}=u_{0} \\
& \left\{\begin{array}{c}
\sigma_{1,1}=u_{1,1}^{1} T_{1}+u_{1,1}^{2} T_{2}+\cdots \\
\cdots \cdots \cdots \cdots \cdots \cdots \cdots \cdots \\
\sigma_{1, a_{1}}=u_{1, a_{1}}^{1} T_{1}+u_{1, a_{1}}^{2} T_{2}+\cdots
\end{array}\right. \\
& \left\{\begin{array}{c}
\sigma_{i, 1}=u_{i, 1}^{i} T_{i}+u_{i, 1}^{i+1} T_{i+1}+\cdots \\
\cdots \cdots \cdots \cdots \cdots \cdots \cdots \cdots \cdots \cdots \\
\sigma_{i, a_{i}}=u_{i, a_{i}}^{i} T_{i}+\dot{u}_{i, a_{i}}^{i+1} T_{i+1}+\cdots
\end{array}\right.
\end{aligned}
$$

where $\left(u_{i, 1}^{i}, u_{i, 2}^{i}, \cdots, u_{i, a_{i}}^{i}\right)$ are contained in $k(\boldsymbol{P})$ and are linearly independent over $k$. Then $\omega$ has the following expression

$$
\begin{aligned}
\omega=\sigma_{0} \tau_{0}+\sigma_{1,1} \tau_{1,1}+\cdots & +\sigma_{1, a_{1}} \tau_{1, a_{1}}+\cdots+\sigma_{i, 1} \tau_{i, 1}+\cdots+\sigma_{i, a_{i}} \tau_{i, a_{i}}+\cdots \\
=u_{0} \tau_{0} \pm\left(u_{1,1}^{1} \tau_{1,1}+\cdots\right. & \left.+u_{1, a_{1}}^{1} \tau_{1, a_{1}}\right) T_{1} \pm \cdots \\
& \pm\left(u_{1,1}^{i} \tau_{1,1}+\cdots+u_{i, 1}^{i} \tau_{i, 1}+\cdots+u_{i, a i}^{i} \tau_{i, a i}\right) T_{i}+\cdots
\end{aligned}
$$

Since the differential forms $u_{1,1}^{i} \tau_{1,1}+\cdots+u_{i, 1}^{i} \tau_{i, 1}+\cdots+u_{i, a_{i}}^{i} \tau_{i, a_{i}}$ on $\boldsymbol{V}$ are of the first kind, by the proposition 10 in $[K]$, we may show by the mathematical induction that $\tau_{i, j}$ are of the first kind. q. e. d.

2) It is not necessary for this theorem for $\boldsymbol{U}$ to have a complete model without singularities, which is birationally equivalent to $\boldsymbol{U}$ over $k$. From the theory of normal variaties we may easily deduce that the theorem holds true if $k$ in perfect. 
2. Next, we consider an extension of the theorem 2 in $[K]$. We begin with the following definition.

DERINITION 1. Let $\omega$ be a differential form on a Variety. U. $\boldsymbol{\omega}$ and $\boldsymbol{U}$ are defined over $a$ field $k$ and $\boldsymbol{P}$ is a generic Point of $\boldsymbol{U}$ over $k$. We say that $\boldsymbol{\omega}$ has the property $(F)$ at a point $\boldsymbol{P}^{\prime}$ on $\boldsymbol{U}$, if $\boldsymbol{\omega}$ has the following expression

$$
\omega=\sum u_{i} d v_{i},
$$

where $u_{i}$ and $v_{i}$ are contained in the specialization ring of $\boldsymbol{P}^{\prime}$ in $k(\boldsymbol{P})$

It is evident that the property $(F)$ at $\boldsymbol{P}^{\prime}$ is equivalent to the finiteness at $\boldsymbol{P}^{\prime}$, when $\boldsymbol{P}^{\prime}$ is a simple Point on $\boldsymbol{U}$.

PROPOSITION 1. Let $\omega$ be a differential form on a complete Variety $\boldsymbol{U}$. If $\omega$ has the property $(F)$ everywhere on $\boldsymbol{U}, \omega$ is of the first kind.

- PROOP. Let $V^{n}$ be a birationally equivalent variety over $k$ to $\boldsymbol{U}$, and $P$ be a simple $(n-1)$-dimensional point over $k$ on $V$. If $\boldsymbol{Q}$ on $\boldsymbol{U}$ is a birationally corresponding Point to $P$, the specialization ring of $P$ includes that of $\boldsymbol{Q}$. Therefore if $\boldsymbol{\omega}$ has the property $(F)$ at $\boldsymbol{Q}$ on $\boldsymbol{U}$, it has the same property at $P$ on $V$. This proves the proposition.

PROPOSITION 2. Let $\boldsymbol{V}$ be a simple Subvariety of $\boldsymbol{U}$ and $\boldsymbol{P}$ be a point on $\boldsymbol{V}$. If a differential form $\boldsymbol{\omega}$ on $\boldsymbol{U}$ is finite on $\boldsymbol{V}$, it induces a differential form $\boldsymbol{\omega}^{\prime}$ on $\boldsymbol{V}$. Moreover, if $\boldsymbol{\omega}$ has the property $(F)$ at $\boldsymbol{P}$ on $\boldsymbol{U}$, $\boldsymbol{\omega}^{\prime}$ has also the same property at $\boldsymbol{P}$ on $\boldsymbol{V}$.

PROOF: The first assertion follows from the proposition 6 in $[K]$. Let $k$ be a common field of definition for $\boldsymbol{U}, \boldsymbol{V}$ and $\omega$, and $\overline{\boldsymbol{P}}, \overline{\boldsymbol{Q}}$ be, respectively, the generic Points of $\boldsymbol{U}, \boldsymbol{V}$ over $k$. Every element in the specialization ring of $\boldsymbol{P}$ in $k(\overline{\boldsymbol{P}})$ has a uniquely determined specialization over $\overline{\boldsymbol{P}} \rightarrow \overline{\boldsymbol{Q}}$ with respect to $k$, and that specialization is contained in the specialization ring of $\boldsymbol{P}$ in $k(\overline{\boldsymbol{Q}})$. This proves the proposition.

From these two propositions we can obtain at once the following theorem.

THEOREM 2. Let $\boldsymbol{U}$ be a complete Variety without singularities, and $\boldsymbol{V}$ be a simple Subvariety of $\boldsymbol{U}$. If a differential form $\boldsymbol{\omega}$ on $\boldsymbol{U}$ is of the first kind, it induces a differential form $\boldsymbol{\omega}^{\prime}$ on $\boldsymbol{V}$ of the first kind.

Mathematical Institute, Nagoya University. 